\title{
EVIDENCE IMPLICATING SUBSTANTIA NIGRA IN REGULATION OF KINDLED SEIZURE THRESHOLD ${ }^{1}$
}

\author{
JAMES O. MCNAMARA, ${ }^{2}$ MARC T. GALLOWAY, LEWIS C. RIGSBEE, AND CHEOLSU SHIN \\ Departments of Medicine (Neurology) and Pharmacology, Duke University Medical Center and Epilepsy Research Laboratory, \\ Veterans Administration Medical Center, Durham, North Carolina 27705
}

Received January 4, 1984; Revised March 6, 1984; Accepted March 21, 1984

\begin{abstract}
We studied the effects of microinjected drugs and brainstem lesions on motor and limbic seizures in the kindling model of epilepsy. The duration of motor seizures was determined by timing the clonic and tonic movements of the extremities. The duration of limbic seizures was determined by measuring afterdischarge recorded on the electroencephalogram.

Bilateral microinjection of a $\gamma$-aminobutyric acid (GABA) agonist, muscimol, into the area of the substantia nigra (SN) markedly suppressed both motor and limbic seizures induced by stimulation of amygdala, olfactory structures, or lateral entorhinal cortex. Microinjection of saline did not suppress seizures.

The suppressive effect of muscimol: (i) dissipated after several hours and was dependent on dose; (ii) was due to an elevation of the seizure threshold, since typical seizures could be elicited with electrical current far exceeding the threshold; and (iii) exhibited spatial specificity since muscimol injections 1 to $2 \mathrm{~mm}$ dorsal to the $\mathrm{SN}$ or into neocortex did not suppress the seizures.

The actions of muscimol were probably mediated by its GABA agonist properties, since microinjection of an irreversible inhibitor of GABA transaminase ( $\gamma$-vinyl GABA) into the area of the SN also suppressed kindled seizures.

Destruction of brainstem structures was produced by microinjection of the neurotoxin, $N$-methyl-D,Laspartate. Seizures were markedly suppressed in animals with bilateral destruction of the SN but not in animals in which the SN was spared bilaterally.

We interpret the data to indicate that the SN is the site at which the GABA agonists and lesions act to raise the threshold for kindled seizures. The suppression of limbic seizures indicates that this brainstem nucleus can regulate the intrinsic neuronal excitability of hemispheric sites.
\end{abstract}

Kindling, an animal model of temporal lobe epilepsy, is a phenomenon in which repeated administration of an initially subconvulsive electrical stimulus eventually results in intense limbic and generalized motor seizures (Goddard et al., 1969). Once established, the kindling effect is permanent.

Anatomic delineation of the underlying neural network is a necessary first step to elucidating the basic cellular and molecular mechanisms of kindling. Myslobodsky et al. (1979) found that systemic administration of $\gamma$-vinyl $\gamma$-aminobutyric acid ( $\gamma$-vinyl GABA) (GVG), a GABA transaminase inhibitor, blocked the motor component of kindled seizures. Iadarola and Gale (1982) found that the substantia nigra (SN) appears to be a key site of GABA-mediated anticonvulsant action, since microinjection of muscimol, an agonist of the inhibitory neu-

${ }^{1}$ This work was supported by National Institutes of Health Grants NS 17771 and NS 16431 and a grant from the Veterans Administration. We wish to thank Ms. Eloise Pittman and Ms. Rena Wethington for their help in preparing the manuscript.

${ }^{2}$ To whom correspondence should be addressed, at Epilepsy Research Laboratory, Veterans Administration Medical Center, 508 Fulton Street, Durham NC 27705. rotransmitter GABA, into the $\mathrm{SN}$ decreased susceptibility to seizures produced by electroshock and chemoconvulsants. Therefore, we hypothesized that the $\mathrm{SN}$ may also regulate the expression of motor seizures in the kindling model.

We report here the results of pharmacologic and lesion experiments which test this hypothesis. A preliminary report of some of these results has been published (McNamara et al., 1983).

\section{Materials and Methods}

Kindled seizure terminology. The behavioral responses to electrical stimulation of amygdala or other sites were graded according to the five classes defined by Racine (1972): (1) facial clonus; (2) head nodding; (3) forelimb clonus; (4) rearing; and (5) rearing and falling. The term "motor seizure" will be used to refer to the duration of clonic or tonic activity of the extremities during a class 4 or 5 seizure. The term "limbic seizure" will be used to refer to behavior in which the rat is immobilized with or without associated facial clonus or head nodding; these behaviors were defined as limbic seizure when correlated with focal afterdischarge recorded between the tips of the bipolar electrode in the amygdala. Seizures manifested by these behaviors are correlated with afterdischarge (Wada and Sato, 1974) and increased 2-deoxyglucose uptake in limbic structures (Engel et al., 1978).

Our experimental paradigms exclusively used animals which con- 
sistently exhibited class 4 or 5 seizures. The typical sequence of seizure expression in these animals consisted of limbic seizure activity for approximately 5 sec prior to a motor seizure which in turn lasted approximately $35 \mathrm{sec}$. Limbic seizure behavior was once again apparent immediately upon termination of motor seizure and typically lasted approximately $35 \mathrm{sec}$. Limbic seizure was quantitated by measuring the duration of afterdischarge recorded from the bipolar electrode in amygdala (Fig. 1). Motor seizure duration was measured by monitoring the clonic or tonic activities of the extremities.

Preparation of animals for microinjection experiments. Male SpragueDawley rats (Charles River Breeding Laboratories) were stereotaxically implanted with a bipolar electrode (twisted nichrome wire, $0.254 \mathrm{~mm}$ diameter) in the right amygdala under pentobarbital anesthesia (McNamara, 1978). Stainless steel guide cannulas (22 gauge, Plastic Products) were implanted and directed toward the injection sites. Electrode assembly and guide cannulas were secured to the skull with dental acrylic.

Animals were maintained thereafter on a light (7:00 AM to 7:00 PM) and dark (7:00 PM to 7:00 AM) cycle. After a postoperative recovery period of at least 7 days, constant current stimulations (200- to 1,000 $\mu \mathrm{A}$ biphasic square wave pulses, each $1 \mathrm{msec}$ in duration, $60 \mathrm{~Hz}$ for 1 sec from a Grass 88 stimulator) were delivered to the amygdala at intervals of at least 90 min until several class 4 or 5 seizures were clicited. Thereafter, stimulations were administered once each day between 12:00 noon and 5:00 PM. The EEG before and after each stimulation was recorded between the tips of the bipolar electrode and from each tip to a referential electrode secured to skull screws. The amount of current delivered during each stimulation was monitored on a Tektronix Type 502A oscilloscope by measuring the voltage drop across a $10,000-\mathrm{ohm}$ resistor in series with the animal.

Daily stimulations were continued until a stable generalized seizure threshold was obtained by determining the responses to specified current intensity on four consecutive days. A stable generalized seizure threshold was defined as a current intensity at which stimulation 20 $\mu \mathrm{A}$ above this value resulted in class 4 or 5 seizures while stimulation $20 \mu \mathrm{A}$ below this value did not. Stable generalized seizure thresholds ranged from 200 to $900 \mu \mathrm{A}$ and were not significantly different among experimental and control groups. Drugs were microinjected 1 day after a stable generalized seizure threshold was established. The effect of the drug was determined by measuring the response to a test stimulus which consisted of current exceeding the generalized seizure threshold by $10 \%$.

Microinjection of drugs. Drugs were microinjected via injection cannulas (28 gauge, Plastic Products) inserted through guide cannulas in awake, gently restrained rats. All injections were made bilaterally. Either saline or drugs dissolved in saline were injected in a volume of $0.5 \mu \mathrm{l}$ at a rate of $0.2 \mu \mathrm{l} / \mathrm{min}$ with a Harvard infusion pump. The injection cannula was withdrawn 2 min after completion of the injection. The animals were carefully observed for behavioral consequences of drug injection between the termination of the injection and stimulation. The interval between termination of injection and stimulation was $30 \mathrm{~min}$ unless specified otherwise.

Some of the animals in the muscimol experiments summarized in Tables I and III received multiple injections. The stable generalized seizure threshold was always re-established between injections in these animals. Some of these animals received muscimol through injection cannulas of different lengths which delivered the drug to different depths of the brainstem. Other animals received saline or muscimol through cannulas of identical lengths. The seizure-suppressant effects of muscimol were evident even after four injections in the same animal. Nonetheless, the results were confirmed in additional animals receiving single injections. Since equivalent results were obtained after single or multiple injections, the data were pooled for presentation.

The paradigm for experiments assessing the dose dependency of the muscimol effect (Table II) differed from that described above. The same three animals received either 10 or $50 \mathrm{ng}$ of muscimol with re-
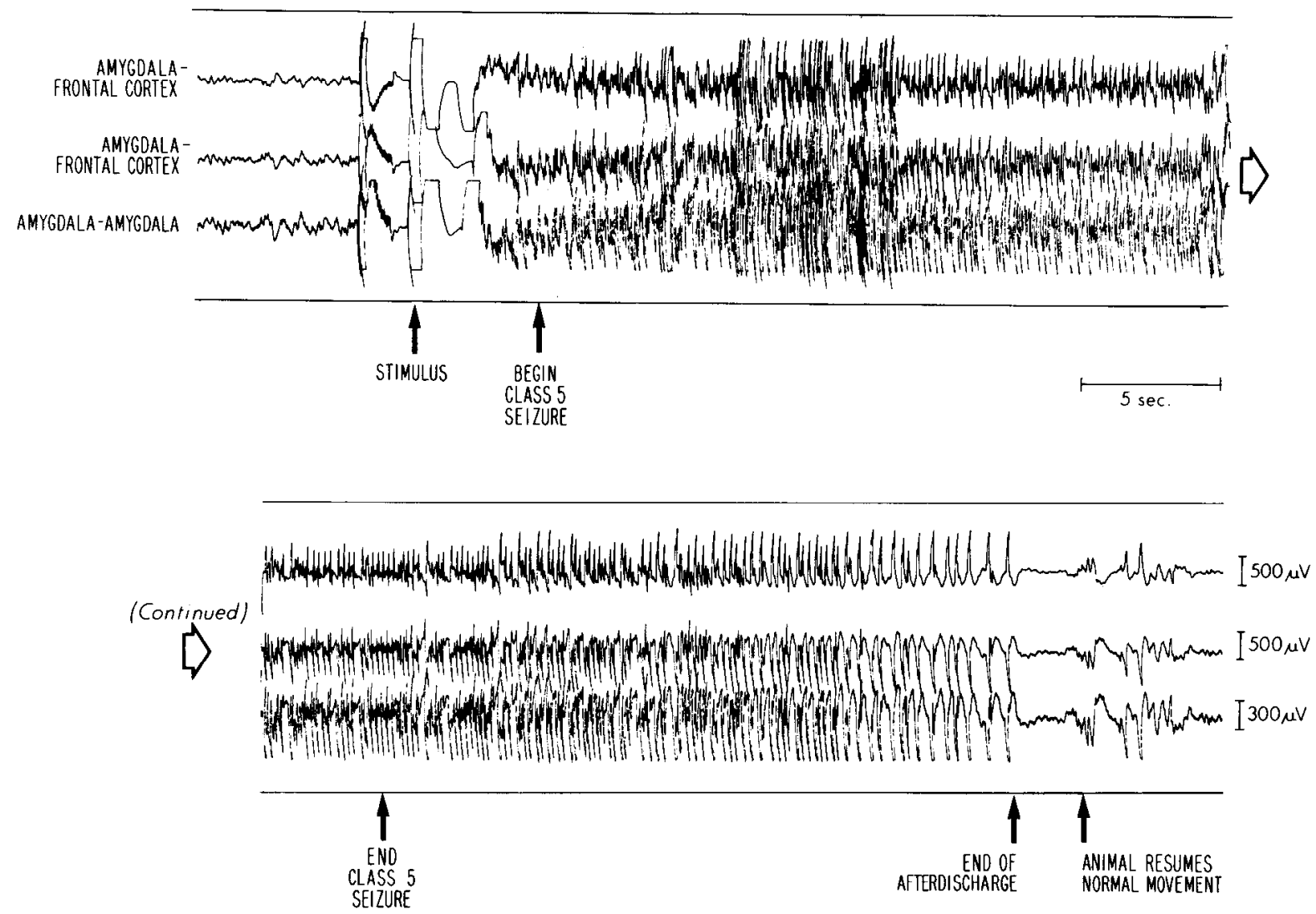

Figure 1. Typical EEG patterns observed during a class 5 seizure. Behaviorally, the animal was immobilized with mild facial clonus for several seconds after cessation of the stimulus prior to exhibiting the class 5 seizure consisting of rearing, loss of postural tone, and clonic and tonic movements of the extremities. After completion of the clonic and tonic components of the seizure, the animal regained his normal posture but remained immobilized with facial clonus and head nodding. This persisted for approximately 35 sec and was followed by resumption of normal movement about the cage and frequent "wet-dog shakes." The afterdischarge terminated a few seconds prior to resumption of normal movement. 
TABLE I

Effects of microinjections of muscimol or saline on the duration of amygdaloid kindled seizures

The values represent the mean \pm SEM of seizure duration in seconds. $n$ refers to the number of individuals from which these data were taken.

\begin{tabular}{|c|c|c|c|c|c|}
\hline Drug & Seizure & $\begin{array}{l}\text { Injection } \\
\text { Site }\end{array}$ & $\begin{array}{c}\text { Pretreatment } \\
\text { Day }\end{array}$ & $\begin{array}{l}\text { Treatment } \\
\text { Day }\end{array}$ & $\begin{array}{c}\text { Post-treatment } \\
\text { Day }\end{array}$ \\
\hline \multirow[t]{2}{*}{ Muscimol (50 ng) } & $\begin{array}{l}\text { Motor seizure } \\
(n=8)\end{array}$ & SN & $34 \pm 3$ & $3 \pm 3^{a}$ & $31 \pm 4$ \\
\hline & $\begin{array}{l}\text { Afterdischarge } \\
(n=4)^{b}\end{array}$ & & $70 \pm 7$ & $10 \pm 8^{a}$ & $66 \pm 10$ \\
\hline \multirow[t]{2}{*}{ Saline } & $\begin{array}{l}\text { Motor seizure } \\
(n=4)\end{array}$ & $\mathrm{SN}$ & $39 \pm 5$ & $39 \pm 8$ & $36 \pm 4$ \\
\hline & $\begin{array}{l}\text { Afterdischarge } \\
(n=2)^{b}\end{array}$ & & $69 \pm 0$ & $64 \pm 2$ & $55 \pm 0$ \\
\hline \multirow[t]{2}{*}{$\begin{array}{l}\text { Muscimol (50 or } 100 \\
\text { ng) }\end{array}$} & $\begin{array}{l}\text { Motor seizure } \\
(n=5)\end{array}$ & Dorsal to SN & $38 \pm 4$ & $27 \pm 3$ & $35 \pm 4$ \\
\hline & $\begin{array}{l}\text { Afterdischarge } \\
(n=4)^{b}\end{array}$ & & $65 \pm 11$ & $55 \pm 15$ & $62 \pm 6$ \\
\hline Muscimol (50 ng) & $\begin{array}{l}\text { Motor seizure } \\
(n=8)\end{array}$ & Neocortex & $36 \pm 3$ & $28 \pm 7$ & $36 \pm 4$ \\
\hline
\end{tabular}

${ }^{a}$ Data from muscimol injections into the region of the $\mathrm{SN}$ are significantly different (Student's $t$ test, two-tailed, $p<0.001$ ) from pretreatment days.

${ }^{b}$ Discrepancy in $n$ between motor seizure and afterdischarge is because data on afterdischarge were not available in all animals due to dislodging of recording electrodes during intense motor seizures.

establishment of a stable generalized seizure threshold between injections. Since the goal was to examine the effect of different doses of the same drug in the same animal, the results were not confirmed in animals receiving a single injection.

Four of the amygdaloid kindled animals receiving microinjections of GVG had previously received injections of muscimol. Similar results were obtained in an additional animal receiving GVG alone, and thus these data were also pooled for presentation.

Neurotoxin lesions. These animals were prepared according to the same protocol as outlined for microinjection experiments. Following establishment of a stable generalized seizure threshold, anesthesia was induced with pentobarbital $(60 \mathrm{mg} / \mathrm{kg})$, and $20 \mu \mathrm{g}$ of $N$-methyl-D,Laspartate in $0.5 \mu \mathrm{l}$ of saline were injected at a rate of $0.2 \mu \mathrm{l} / \mathrm{min}$ through injection cannulas inserted through guide cannulas. The injection cannula was withdrawn 5 min after completion of the injection. All injections were made bilaterally. The depth of the injection was adjusted in individual animals by using injection cannulas which extended beyond the tip of the guide cannula by varying distances. Three days later the animals received a test stimulation at current intensity $10 \%$ above the generalized seizure threshold. Following completion of the experiment, the animals were sacrificed and the extent of the lesion was reconstructed from Nissl stains of scrial coronal sections. The reconstructions were done by one of the authors (J. O. M.) without prior knowledge of the seizure responses after lesions.

Stereotaxic coordinates. Coordinales for the electrodes were: amygdala, $0.8 \mathrm{~mm}$ posterior to bregma, $4.8 \mathrm{~mm}$ right of sagittal suture, and $8.5 \mathrm{~mm}$ below dura; olfactory structures, $6.1 \mathrm{~mm}$ anterior to bregma, $1.2 \mathrm{~mm}$ right of sagittal suture, and $4.5 \mathrm{~mm}$ below dura; and lateral entorhinal cortex, $3.4 \mathrm{~mm}$ anterior to the tip of the transverse sinus, $0.6 \mathrm{~mm}$ right of sagittal suture, and $6.0 \mathrm{~mm}$ below dura at an angle $30^{\circ}$ from the sagittal plane. Coordinates for guide cannulas were: overlying $\mathrm{SN}, 3.4 \mathrm{~mm}$ posterior to bregma, $2.1 \mathrm{~mm}$ on either side of sagittal suture, and $2.5 \mathrm{~mm}$ below dura: anterior neocortex, $3.0 \mathrm{~mm}$ anterior to bregma, $2.5 \mathrm{~mm}$ on either side of sagittal suture, and $2.0 \mathrm{~mm}$ below dura; posterior neocortex, $0.2 \mathrm{~mm}$ posterior to bregma, $1.5 \mathrm{~mm}$ on either side of sagittal suture, and $2.0 \mathrm{~mm}$ below dura.

Histology. Placements of stimulating electrodes and injection cannula tips were verified histologically in all animals. Animals were sacrificed by decapitation. The brains were removed rapidly and frozen in isopentane chilled in a dry ice/methanol bath. Sixteen-micrometerthick coronal frozen sections were taken serially and Nissl-stained sections were analyzed.

Drugs. Muscimol and $N$-methyl-D,L-aspartate were obtained from Sigma Chemical Co. (St. Louis, MO). $\gamma$-vinyl GABA was a gift from Merrell Laboratories (Cincinnati, $\mathrm{OH}$ ).

Statistics. Means and SEM were calculated. Significance of differences was assessed from the Student's two-tailed $t$ test.

\section{Results}

Effects of muscimol microinjections on amygdaloid kindled seizures. Bilateral microinjections of muscimol $(50 \mathrm{ng} / \mathrm{side})$ into or adjacent to the SN markedly suppressed motor seizures (Fig. 2 , top, and Table I). The $91 \%$ suppression was highly significant $(p<0.001)$ in comparison to either the day preceding or the day following the injection. Motor seizures were abolished in seven of the eight animals. Microinjection of saline into equivalent areas did not suppress motor seizures (Fig. 2, middle, and Table I).

Interestingly, muscimol microinjections into the region of the SN also suppressed limbic seizures. This was evident from both behavioral and EEG observations. The $85 \%$ suppression of afterdischarge duration in these animals was statistically significant (Table 1 and Fig. 2, top). Data on afterdischarge duration are presented in Table I in only four of the eight animals receiving muscimol in the region of the $\mathrm{SN}$; the reason for excluding four of the animals was that the data on afterdischarge were not available on pretreatment or post-treatment day because recording electrodes were dislodged during intense motor seizures. The data were available for all eight of these animals on the day of muscimol treatment because motor seizures were suppressed. The afterdischarge duration (mean \pm SEM) was $17 \pm 8 \mathrm{sec}$ which is not significantly different from the value of $10 \pm 8 \mathrm{sec}$ reported for the four animals in Table I. Four of these eight animals exhibited no afterdischarge at all. In contrast to these results, no statistically significant depression of afterdischarge duration was observed after saline injection into the area of the $\mathrm{SN}$ or after muscimol injection into brainstem sites dorsal to the area of the SN (Table I and Fig. 2, middle and bottom).

The seizure-suppressant effect exhibited spatial specificity, since microinjection of $50 \mathrm{ng}$ (three animals) or $100 \mathrm{ng}$ (two animals) of muscimol in sites 1 to $2 \mathrm{~mm}$ dorsal to the $\mathrm{SN}$ did not significantly reduce motor seizure or afterdischarge duration (Fig. 2, bottom, and Table I). Bilateral microinjections of muscimol into areas of neocortex of eight animals $(50 \mathrm{ng} / \mathrm{side}$ in seven animals, $100 \mathrm{ng} /$ side in one animal) did not significantly attenuate motor seizure duration (Table I).

To determine whether the seizure-suppressant action of muscimol was dose dependent, three kindled animals received injections of 10 or $50 \mathrm{ng}$ on separate days, and the effects of these 


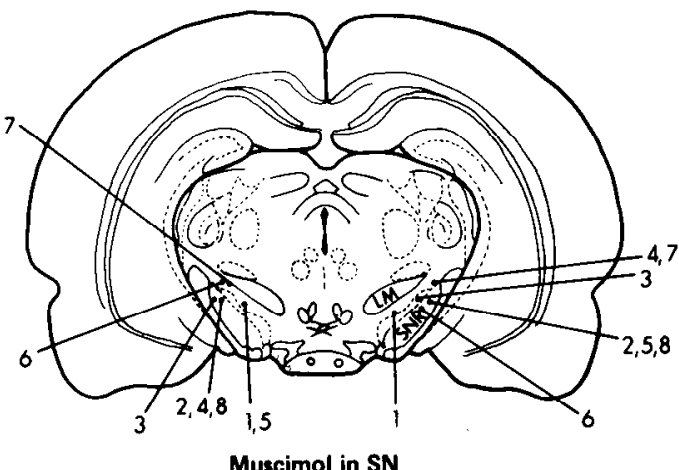

Muscimol in SN

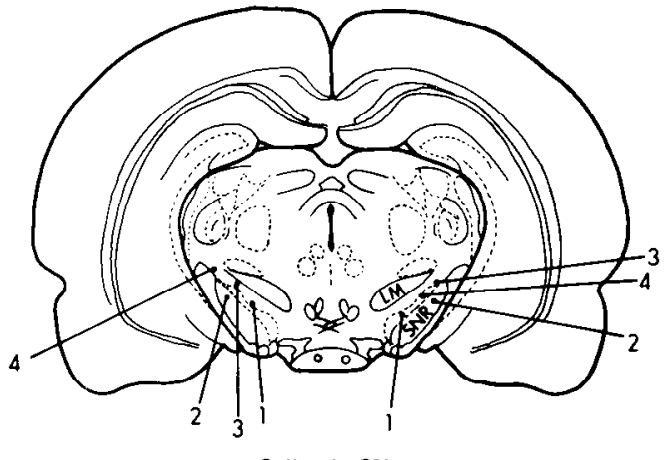

Saline in SN

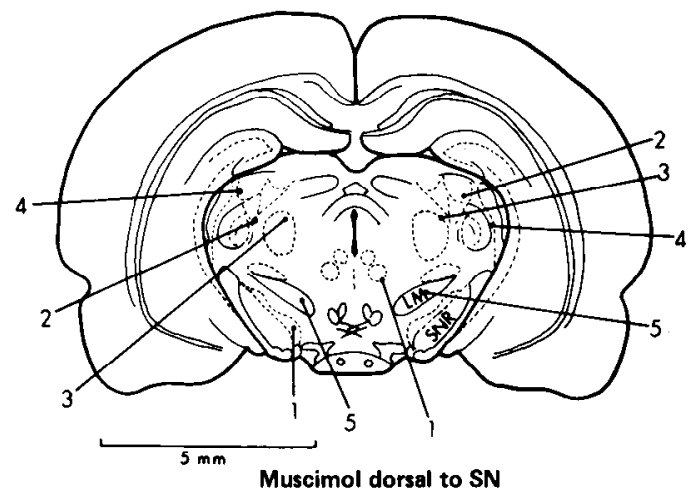

Figure 2. Locations of injection cannula tips plotted on the drawings of coronal sections through the brainstem according to the stereotaxic atlas of Konig and Klippel (1963). The right side of the brain corresponds to the right side of the figure. Structures in this figure are identified in Figure 5. All animals received bilateral injections. The sites of injection cannula tips, as determined by examination of Nisslstained serial frozen sections, are indicated by small dots. The number corresponding to each dot refers to the left and right sides of a single animal from each group. The rostral and caudal extent of locations of cannula tips ranged approximately $500 \mu \mathrm{m}$ on either side of this level with the exception of animal 1 (bottom); the cannula on the right side of this animal was situated $1.2 \mathrm{~mm}$ rostral to this level. The data from these animals are presented in Table I. $L M$, medial lemniscus; SNR, substantia nigra, pars reticulata.

doses were compared in the same animals (Table II). Suppression of both motor seizure and afterdischarge was obtained with both doses, but statistically significant effects were obtained only with the 50 -ng dose.

Effects of muscimol microinjections on kindled seizures elicited from sites outside amygdala. To determine whether the seizuresuppressant action of muscimol was unique to kindled seizures elicited from amygdala, we examined the effects of muscimol in animals kindled from lateral entorhinal cortex or olfactory structures (Table III). Microinjection of muscimol into the region of the $\mathrm{SN}$ abolished motor seizures elicited from lateral entorhinal cortex (Fig. 3 , bottom) and produced an $87 \%$ reduction in duration of motor seizures elicited from olfactory structures (Fig. 3, top). Afterdischarges elicited from these sites were also dramatically attenuated. These suppressive effects were no longer detectable $24 \mathrm{hr}$ following injection.

Additional evidence for the spatial specificity of the muscimol effect was obtained from three animals kindled from olfactory sites. At least one of the injection sites in each of these animals was situated dorsal or lateral to the SN (Fig. 3, middle). 'I'hese injections resulted in reductions of $33 \%$ and $38 \%$ of motor seizure and afterdischarge duration, respectively. This partial suppression was considerably less marked than that obtained with injections more directly involving the $\mathrm{SN}$ (Fig. 3, top).

TABLE II

Dose dependency of muscimol effect on the duration of amygdaloid kindled seizures

Values represent the mean \pm SEM of seizure duration in seconds. Data were obtained from three animals.

\begin{tabular}{clccc}
\hline Dose & Seizure & $\begin{array}{c}\text { Pretreatment } \\
\text { Day }\end{array}$ & $\begin{array}{c}\text { Treatment } \\
\text { Day }\end{array}$ & $\begin{array}{c}\text { Post-treatment } \\
\text { Day }\end{array}$ \\
\hline \multirow{2}{*}{$10 \mathrm{ng}$} & Motor Seizure & $38 \pm 6$ & $11 \pm 11$ & $35 \pm 4$ \\
& Afterdischarge & $62 \pm 6$ & $32 \pm 22$ & $64 \pm 17$ \\
$50 \mathrm{ng}$ & Motor Seizure & $44 \pm 10$ & $0^{a}$ & $31 \pm 5$ \\
& Afterdischarge & $92 \pm 24$ & $15 \pm 10^{b}$ & $62 \pm 6$ \\
\hline
\end{tabular}

${ }^{a} p<0.02$, Student's $t$ test, two-tailed.

${ }^{b} p<0.05$, Student's $t$ test, two-tailed.

\section{TABLE III}

Effects of microinjections of muscimol on the duration of kindled seizures elicited from lateral entorhinal cortex or olfactory structures

Values represent the mean \pm SEM of seizure duration in seconds. $n$ refers to the number of individual animals from which these data were taken.

\begin{tabular}{ccccc}
\hline $\begin{array}{c}\text { Brain Structure } \\
\text { and Seizure }\end{array}$ & $\begin{array}{c}\text { Injection } \\
\text { Site }\end{array}$ & $\begin{array}{c}\text { Pretreatment } \\
\text { Day }\end{array}$ & $\begin{array}{c}\text { Treatment } \\
\text { Day }\end{array}$ & $\begin{array}{c}\text { Post-treatment } \\
\text { Day }\end{array}$ \\
\hline $\begin{array}{c}\text { Lateral entorhinal } \\
\text { cortcx }\end{array}$ & & & & \\
$\begin{array}{c}\text { Motor Seizure } \\
(n=3)\end{array}$ & $\mathrm{SN}$ & $41 \pm 4$ & $0^{a}$ & $31 \pm 4$ \\
$\begin{array}{c}\text { Afterdischarge } \\
\quad(n=3)\end{array}$ & & $83 \pm 11$ & $1 \pm 1^{a}$ & $106 \pm 16$ \\
$\begin{array}{c}\text { Olfactory structures } \\
\text { Motor Seizure } \\
\quad(n=6)\end{array}$ & $\mathrm{SN}$ & $38 \pm 4$ & $5 \pm 3^{a}$ & $39 \pm 2$ \\
$\begin{array}{c}\text { Afterdischarge } \\
\quad(n=3)^{c}\end{array}$ & & $79 \pm 9$ & $14 \pm 13^{d}$ & $67 \pm 13$ \\
$\begin{array}{c}\text { Olfactory structures } \\
\text { Motor Seizure } \\
(n=3)\end{array}$ & Dorsal or & $36 \pm 2$ & $24 \pm 3$ & $31 \pm 3$ \\
$\quad \begin{array}{llll}\text { lateral } \\
\text { Afterdischarge } \\
(n=3)\end{array}$ & to SN & & & \\
\hline
\end{tabular}

${ }^{a} p<0.005$, Student's $t$ test, two-tailed.

${ }^{b}$ The electrode tips in these animals were situated in the olfactory bulb ( $n=2)$, olfactory tract $(n=2)$, or anterior olfactory nucleus ( $n=$ 2). The data were grouped for presentation because comparable results were obtained from each site and the unknown extent of electrical current spread raises question as to the validity of separating them.

' Three of six animals in this group dislodged the recording electrodes during motor seizures not allowing measurements of afterdischarge.

${ }^{d} p<0.02$, Student's $t$ test, two-tailed. 
Duration of seizure-suppressant action of muscimol. To assess the duration of action of muscimol, five animals which exhibited seizure suppression $30 \mathrm{~min}$ after muscimol injection received additional stimulations identical to the test stimulus at $30-, 60-$, or 90 -min intervals for up to $5 \mathrm{hr}$ and another at 24 hr after muscimol injection. The muscimol effect disappeared in four of the animals between 2 and $5 \mathrm{hr}$ after injection. A fifth animal responded with only brief (less than $13 \mathrm{sec}$ ) afterdischarges at 30 -min intervals for $5 \mathrm{hr}$; a response equivalent to the pretreatment seizure occurred $24 \mathrm{hr}$ after injection. Iimbic seizure activity returned before motor seizure activity in all five animals. These five as well as all other muscimol-

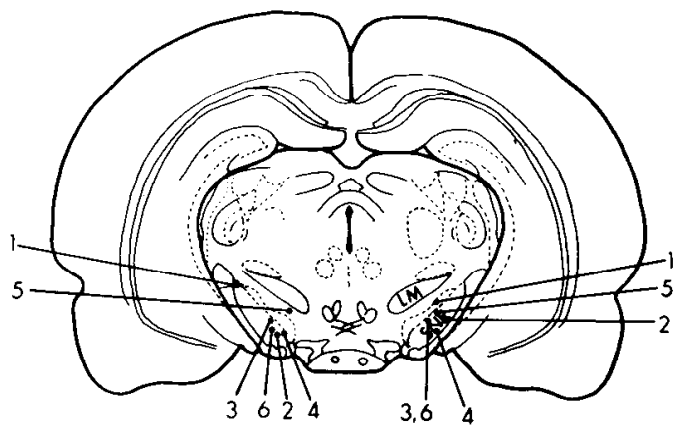

Olfactory kindled/muscimol in SN

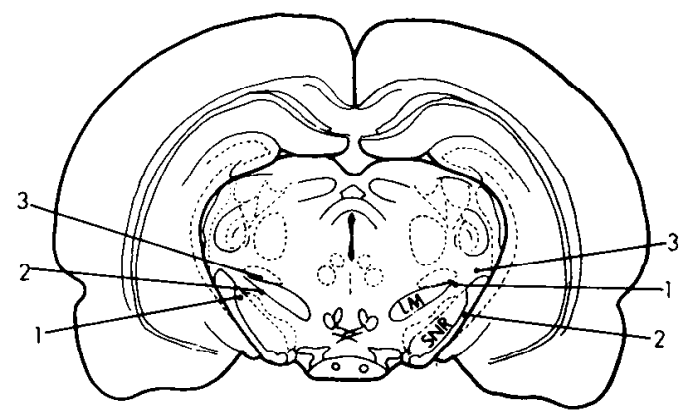

Olfactory kindled/muscimol dorsolateral to SN

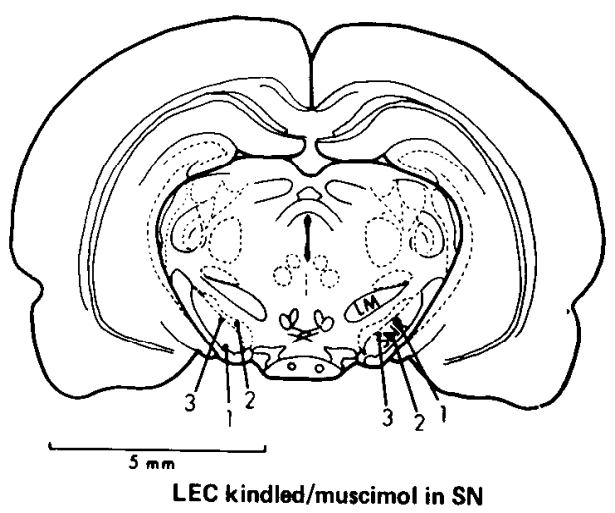

Figure 3. Top, Location of injection cannula tips in animals kindled from olfactory structures with injections in the region of the SN. Middle, Location of injection cannula tips in animals kindled from olfactory structures with injections dorsal and lateral to the SN. Bottom, Location of injection cannula tips in animals kindled from lateral entorhinal cortex. Data from these animals are presented in Table III. $L E C$, lateral entorhinal cortex; $L M$, medial lemniscus; $S N R$, substantia nigra, pars reticulata.

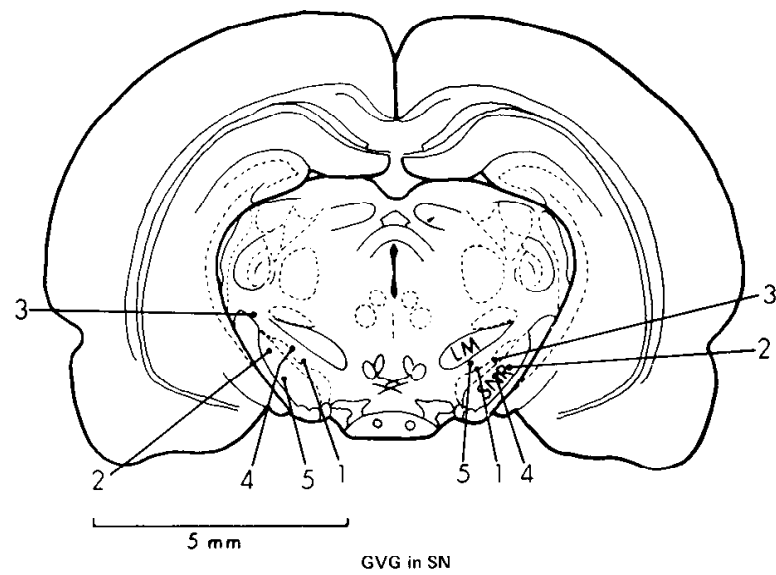

Figure 4. Sites of injection cannula tips in animals receiving GVG as presented in Table IV. $L M$, medial lemniscus; SNR, substantia nigra, pars reticulata.

treated animals exhibited seizures comparable to pretreatment seizures when tested $24 \mathrm{hr}$ after injection.

Effects of suprathreshold stimulation on muscimol suppression. The effect of muscimol consisted of an elevation of seizure threshold, since typical seizures could be elicited with electrical current exceeding the threshold. To assess this effect directly, a series of suprathreshold stimulations was administered to each of five animals (three kindled from olfactory structures and two from amygdala) immediately after an attenuated response had been detected following muscimol treatment. This series consisted of individual 1-sec trains of pulses identical to the test stimuli except that the current of each successive train was increased by approximately $15 \%$ over the preceding train. The interval between each train was approximately $30 \mathrm{sec}$. Three of the animals exhibited typical class 4 or 5 seizures; the remaining two exhibited afterdischarges of 27 and $63 \mathrm{sec}$ which were associated with walking around the cage with occasional forelimb clonus. The mean percentage of increase $( \pm$ SEM) of current over the generalized seizure threshold required to elicit the seizures was $72 \pm 14 \%$ with a range from 38 to $119 \%$. Muscimol suppressed motor seizures more effectively than afterdischarges, since every animal exhibited at least several seconds of afterdischarge at stimulus intensities below those required to elicit motor seizures.

Effect of microinjections of GVG on kindled seizures. GVG is an irreversible inhibitor of GABA transaminase (Lippert et al., 1977). Microinjection of this drug has been demonstrated to increase GABA content in a subcellular fraction of the SN which contains nerve terminals (Iadarola and Gale, 1981). To test the idea that the actions of muscimol were mediated by its GARA agonist properties, GVG was microinjected bilaterally (5 $\mu \mathrm{g} /$ side) into the region of the SN (Fig. 4), and the animals were stimulated from the amygdala at specified intervals until the suppressive effect had dissipated (Table IV). This treatment abolished motor seizures in each of the animals $24 \mathrm{hr}$ after the injection. Afterdischarges were abolished in three of the animals and markedly suppressed in the other two after $24 \mathrm{hr}$. Microinjection of GVG $(5 \mu \mathrm{g} / \mathrm{side})$ bilaterally into the $\mathrm{SN}$ of one animal kindled from the olfactory tract resulted in a time course of seizure suppression similar to that of the amygdaloid kindled group (data not shown). These effects were not due to delayed effects of the injection procedure alone since microinjection of saline into the area of the $\mathrm{SN}$ in two animals did not suppress either afterdischarge or motor seizures elicited by stimulation $24 \mathrm{hr}$ later (data not shown). The latency to onset of the suppressive effect and the time course of its reversal 
TABLE IV

Microinjection of GVG into region of SN: Effects on the duration of amygdaloid kindled seizures

Values represent the mean \pm SEM of seizure duration in seconds. $n$ refers to the number of individual animals from which these data were taken. The number of animals exhibiling these motor seizures is specified in the middle row. Afterdischarge duration was available in only four of five animals because dislodgement of the recording electrodes during the intense motor seizure precluded measurement in one animal.

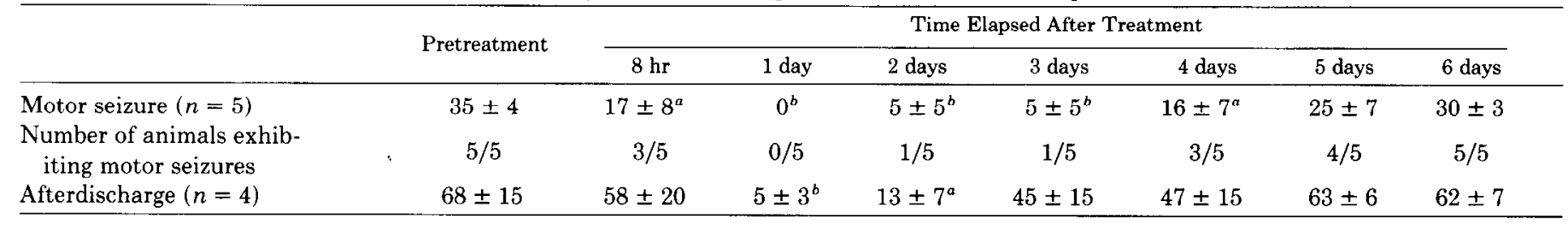

${ }^{a} p<0.05$, Student's $t$ test, two-tailed.

${ }^{b} p<0.01$, Student's $t$ test, two-tailed.

paralleled that of the increased GABA content following microinjection of GVG reported by Iadarola and Gale (1981). The magnitude and duration of motor scizure suppression exceeded that of afterdischarge suppression. We did not examine the effects of microinjections of GVG into other brain regions on amygdaloid kindled seizures. These data support the idea that enhancement of GABA-mediated neurotransmission is the mechanism of seizure suppression.

Effects of lesions on kindled seizures. We postulated that the seizure-suppressant action of muscimol was mediated by reducing neuronal activity in the SN. 'Ihis hypothesis was based on two observations: (1) the spatial selectivity of seizure-suppressing effects of muscimol suggested that the SN was the site of action of muscimol; and (2) iontophoretic application of muscimol inhibited unit activity in the SN (Waszczak et al., 1980). This hypothesis predicts that destruction of the SN would suppress kindled seizures.

To test this postulate, we studied the effects of lesions produced with the neurotoxin, $N$-methyl-D,L-aspartate (Nadler et al., 1981), on kindled seizures. We divided the animals into three groups: animals with no SN destruction (group 1), animals with unilateral SN destruction (group 2), and animals with bilateral SN destruction (group 3) (Table V). Reconstructions of the lesions in representative animals from these three groups are depicted in Figure 5. Motor seizures and afterdischarges after the lesion were suppressed by $81 \%$ and $71 \%$, respectively, in animals with bilateral SN destruction compared to prelesion seizure durations. Animals with unilateral SN destruction exhibited suppression of motor seizures $(30 \%)$ and afterdischarges $(32 \%)$ which was less than that with bilateral destruction but exceeded that in which the SN was spared (21\% suppression of motor seizures and $7 \%$ reduction of afterdischarges). The reductions after bilateral lesions were statistically significant $(p<0.001)$, whereas reductions in neither of the other groups were significant $(p>0.05)$.

The destruction of structures other than the SN in these cases warranted careful consideration of the potential role of these structures in seizure suppression. We examined the correlation between bilateral destruction of each structure listed in the legend of Figure 5 and total suppression of motor seizures. Motor seizure suppression correlated best with bilateral destruction of the SN. An effect due to destruction of the reticular formation situated between pars compacta of the SN and the medial lemniscus could not be excluded, since the destruction of this area in the lesion paralleled that of the SN. By contrast, the mamillary body and interpeduncular nucleus could be readily excluded since these structures were spared in all animals with seizure suppression. Destruction of the ventral tegmental area correlated less well with seizure suppression than did destruction of the SN. Among animals in which motor seizures were abolished, the SN was destroyed bilaterally in seven of eight animals, whereas the ventral tegmental area was destroyed bilaterally in five of eight. The results of these analyses

\section{TABLE V}

Effects of lesions on the duration of amygdaloid kindled seizures

Values represent the mean \pm SEM of seizure duration in seconds. Numbers in parenthesis refer to the number of animals. SN was spared in animals in group 1, was at least partially destroyed unilaterally among animals in group 2, and was at least partially destroyed bilaterally among animals in group 3. Data on afterdischarge were available in fewer animals than motor seizure duration (group 1) because one animal dislodged a recording electrode during the seizure. Differences between prelesion and postlesion values were not significant $(p>0.05)$ unless denoted by Footnote $a$.

\begin{tabular}{|c|c|c|c|c|c|c|}
\hline & \multicolumn{2}{|c|}{ Group 1} & \multicolumn{2}{|c|}{ Group 2} & \multicolumn{2}{|c|}{ Group 3} \\
\hline & Prelesion & Postlesion & n Prelesion & Postlesion & Prelesion & Postlesion \\
\hline $\begin{array}{c}\text { Motor seizure } \\
\qquad(n)\end{array}$ & $\begin{array}{c}34 \pm 4 \\
(5)\end{array}$ & $\begin{array}{c}27 \pm 1 \\
(5)\end{array}$ & $\begin{array}{c}37 \pm 3 \\
(4)\end{array}$ & $\begin{array}{c}26 \pm 11 \\
(4)\end{array}$ & $\begin{array}{c}32 \pm 2 \\
(10)\end{array}$ & $\begin{array}{c}6 \pm 3^{a} \\
(10)\end{array}$ \\
\hline $\begin{array}{l}\text { Afterdischarge } \\
\qquad(n)\end{array}$ & $\begin{array}{c}96 \pm 8 \\
(4)\end{array}$ & $\begin{array}{c}89 \pm 12 \\
(4)\end{array}$ & $107 \pm 14$ & $\begin{array}{c}73 \pm 23 \\
(4)\end{array}$ & $\begin{array}{c}89 \pm 5 \\
(10)\end{array}$ & $\begin{array}{c}26 \pm 10^{a} \\
(10)\end{array}$ \\
\hline
\end{tabular}

${ }^{a} p<0.001$, Student's $t$ test, two-tailed.

were consistent with the idea that destruction of the SN was the factor responsible for seizure suppression.

The effect of $\mathrm{SN}$ destruction represented an increase in seizure threshold rather than an elimination of the ability to generate seizures. Additional stimuli equivalent to the test stimulus were administered at intervals of at least $1 \mathrm{hr}$ to three animals in which motor seizures were abolished and brief or no afterdischarges were obtained. None of these subsequent stimuli elicited motor seizures, and the duration of afterdischarge elicited was $20 \mathrm{sec}$ or less. However, administration of an additional stimulus in which both current intensity and train duration were doubled resulted in long afterdischarges in all three animals (98, 70, and $42 \mathrm{sec}$, respectively) accompanied by class 4 seizures in two of the three animals. Extensive destruction of the SN bilaterally was found in all three animals.

Behavioral responses to drugs and lesions. Microinjection of muscimol ( $50 \mathrm{ng}$ ) into the region of the SN produced stereotypies including sniffing, chewing, and some circling behavior. This raised the possibility that the behavioral responses per se may be responsible for the seizure-suppressant effect. To test this idea, stereotypies were induced by systemic administration of apomorphine $(2 \mathrm{mg} / \mathrm{kg}$, i.p.), and the effects on kindled seizures were studied. A generalized seizure threshold was established in four amygdaloid kindlcd animals. These animals were treated with apomorphine and their responses to an electrical stimulus $10 \%$ above the generalized seizure threshold were determined 20 min later. All four animals exhibited chewing and sniffing stereotypies. This treatment did not suppress the seizures. Motor seizure durations (mean \pm SEM in seconds) were: pretreatment, $37 \pm 4$; treatment, $35 \pm 4$; and posttreatment, $40 \pm 5$. Measurement of afterdischarge duration was 


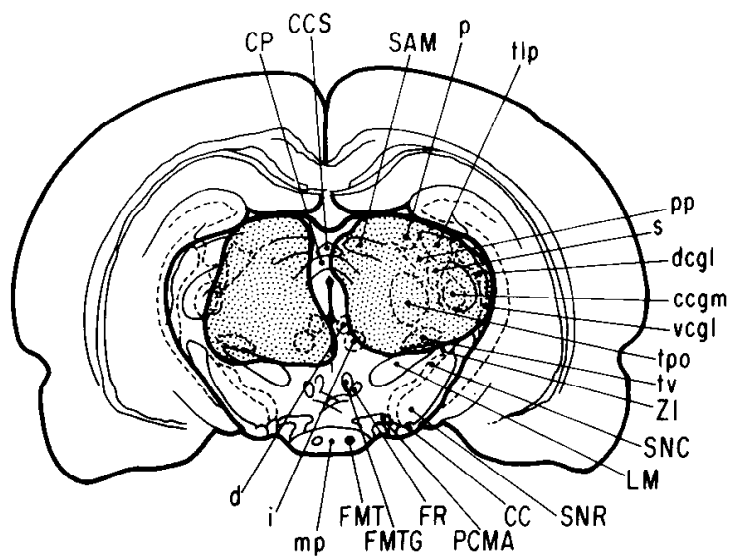

SN spored bilaterally

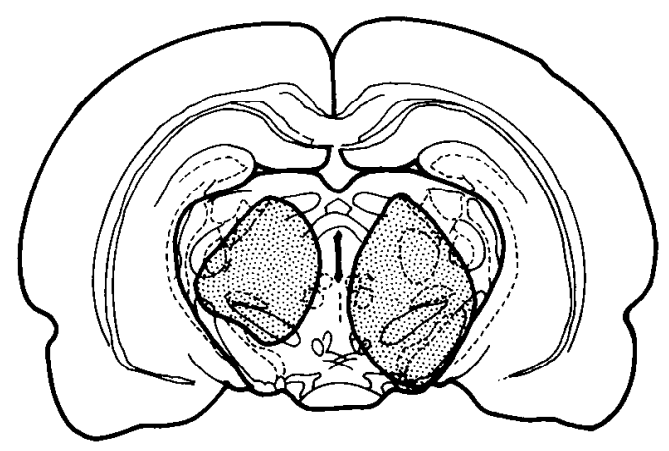

SN destroyed unilaterally

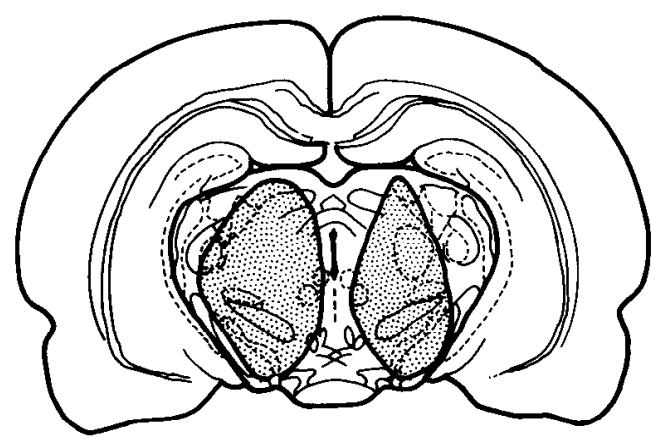

SN destroyed bilaterally

$5 \mathrm{~mm}$

Figure 5. The stippled areas reflect the extent of lesions produced by the neurotoxin, $N$-methyl-D,L-aspartate, in animals presented in Table V. Nissl-stained serial coronal frozen sections were analyzed to determine the extent of the lesions. Only a representative section from each group is presented corresponding to the level A $2420 \mu \mathrm{m}$ according to the stereotaxic atlas of Konig and Klippel (1963). Top, Lesion spared the $\mathrm{SN}$ bilaterally (Table V, group 1). Middle, Lesion destroyed the SN only unilaterally (Table V, group 2). Bottom, Lesion destroyed the SN bilaterally (Table V, group 3). CC, cerebral peduncle; ccgm, central nucleus of medial geniculate; CCS, commissure of the superior colliculus; $C P$, posterior commissure; $d$, nucleus Darkschewitsch; $d c g l$, dorsal nucleus of lateral geniculate; $F M T$, mamillothalamic tract; FMTG, mamillotegmental tract; $F R$, fasciculus retroflexus; $i$, interstitial nucleus of Cajal; $L M$, medial lemniscus; $m p$, posterior mamillary nucleus: $p$, pretectal nucleus; $P C M A$, peduncle of mamillary body; $p p$, pretectal nucleus, pars profundus; $s$, suprageniculate nucleus; $S A M$, stratum album of superior colliculus; $S N C$, substantia nigra, pars compacta; $S N R$, substantia nigra, pars reticulata; $t l p$, lateral thalamic nucleus, pars posterior; tpo, posterior thalamic nucleus; $t v$, ventral thalamic nucleus; $v \mathrm{cgl}$, ventral nucleus of lateral geniculate; $Z I$, zona incerta. available in three of these animals and disclosed: pretreatment, $101 \pm 8$; treatment, $127 \pm 21$; and post-treatment, $94 \pm 15$.

Animals receiving microinjections of GVG exhibited no discernible behavioral alteration, despite the powerful seizuresuppressant effect. Together with the apomorphine results, the data indicate that stereotypies are neither necessary nor sufficient to suppress kindled seizures.

Animals with lesions involving the SN exhibited behavioral abnormalities when examined on the day of test stimulation. All animals with either unilateral or bilateral destruction of the SN exhibited decreased locomotor activity. Chewing stereotypies were observed in half the animals with bilateral lesions but in none with unilateral lesions. Analysis of these behaviors in relation to seizure suppression indicated that reduced locomotor activity was not sufficient to mediate seizure suppression. Moreover, seizure suppression occurred in animals without chewing stereotypies. Thus, these behavioral consequences of the lesion do not appear sufficient to account for the seizure suppression observed.

\section{Discussion}

Two lines of evidence point to the $\mathrm{SN}$ as a crucial site responsible for seizure suppression: (1) the spatial selectivity of microinjections of the GABA agonist, muscimol, together with the fact that the $\mathrm{SN}$ is the principal site of GABA receptors in ventral midbrain, and (2) the correlation between seizure suppression and neurotoxin-mediated destruction of the SN.

Precisely which neuronal constituents within the $\mathrm{SN}$ are responsible for the seizure-suppressant effect is unclear. The dopaminergic neurons of pars compacta are not likely to be involved, since neither dopamine receptor agonists nor antagonists suppress kindled seizures (Babington and Wedeking, 1973). The much greater sensitivity to muscimol of neurons in pars reticulata than in pars compacta (Wasczak et al., 1980) supports pars reticulata as the key site. The pars reticulata also seems more likely in view of its extensive, often bilateral and often collateralized, projections to numerous thalamic and brainstem nuclei (Gerfen et al., 1982; Parent et al., 1983); these connections render it capable of modulating neuronal activity diffusely throughout the brain.

Speculation regarding the role of substantia nigra in kindled seizures. Our results suggest that reduction of neuronal activity within the SN underlies the seizure-suppressant effect. Injection of muscimol, which inhibits SN unit activity (Waszczak et al., 1980), suppresses seizures. Destruction of SN also suppresses seizures. Indeed the presence of afterdischarge recorded from electrodes in the SN during limbic seizures (Wada and Sato, 1974; Wada et al., 1978) raises the possibility that the SN must exhibit increased and/or synchronized neuronal firing in order for seizures to occur.

The suppression of motor seizures is consistent with the idea that the SN transmits seizure information directly from rostral sites of origin to caudal motor targets. Alternatively, the SN could regulate other neuronal structures that propagate the seizure information. The present findings extend previous observations that application of muscimol in the area of the SN suppresses motor seizures induced by electroshock and pentylenetetrazol (Iadarola and Gale, 1982).

The most striking finding of the present work is that focal application of muscimol and GVG to the SN can suppress limbic seizure activity including electrical afterdischarge at the site of stimulation. If the $\mathrm{SN}$ is actively involved in limbic seizure generation, it is not simply transmitting or regulating transmission of seizure information from rostral sites of origin to caudal targets. Rather, this nucleus in the midbrain is regulating the intrinsic neuronal excitability of multiple sites in the cerebral hemispheres including lateral entorhinal cortex, amygdala, and olfactory structures. 
This regulatory influence is almost certainly mediated through polysynaptic pathways, since no direct connections either to or from the $\mathrm{SN}$, on the one hand, and olfactory structures and lateral entorhinal cortex, on the other, have been identified (Gerfen et al., 1982). Whether the interactions between amygdala and the SN are mediated through polysynaptic connections is less clear, since reciprocal connections do exist between the central nucleus of the amygdala and pars lateralis of the SN (Bunney and Aghajanian, 1976; Loughlin and Fallon, 1983).

These findings lead us to propose the following hypothesis to explain how these structures interact to generate a limbic seizure in the kindling model. The train of stimuli activates neurons in the immediate vicinity (e.g., lateral entorhinal cortex) of the electrode. The repetitive firing of these neurons activates targets (e.g., in hippocampal formation and subsequently ventral striatum) which in turn activate neurons in pars reticulata of the $\mathrm{SN}$. Working through polysynaptic connections, the output from the $\mathrm{SN}$ mediates net excitation (either through disinhibition or a direct excitation) of neurons at the site of the stimulating electrode and increases the likelihood of neuronal firing lasting beyond cessation of the stimulation train. Thus reduction of neuronal excitability in the SN would reduce the likelihood of reverberatory activity in the network and thereby raise the seizure threshold.

Relationship to previous hypotheses of seizure generation. Previous investigators have proposed the involvement of subcortical and brainstem structures in the propagation of seizure activity from cortical sites and in the synchronization of hemispheres in seizure activity. Hayashi (1953a, b) suggested that the SN was one component of an efferent pathway mediating propagation of seizures from motor cortex. He based this suggestion on the observation that lesions in rostral midbrain suppress seizures induced by application of nicotine at cortical sites.

Drawing from his neurosurgical experience in epileptic patients, Penfield (1952) proposed a concept of a centrencephalic coordinating and integrating system that is symmetrically related to both hemispheres. The anatomic substrate of this system was proposed to be two areas, of gray matter symmetrically placed in the higher brainstem. He suggested that cortical discharges can lead to generalized convulsions by activation of this centrencephalic system. He further subdivided this system, one part having to do with motor control which became affected during generalized motor seizures. The other part was involved in consciousness or perception and became involved during epileptic automatisms equivalent to limbic seizures (Penfield, 1969).

The present work demonstrates that a specific anatomic structure, the SN, may mediate the functions ascribed to the centrencephalic system. The work further suggests that the SN may be operative not only in the propagation of motor seizure information but also in the generation of limbic seizures at the site of origin. Identifying the nuclei mediating the interactions between limbic structures and the SN will be necessary to determine whether and how these structures interact to generate seizures.

\section{References}

Babington, R. G., and P. W. Wedeking (1973) The pharmacology of seizures induced by sensitization with low intensity brain stimulation. Pharmacol. Biochem. Behav. 1: 461-467.

Bunney, B. S., and G. K. Aghajanian (1976) The precise localization of nigral afferents in the rat as determined by a retrograde tracing technique. Brain Res. 117: 423-435.

Engel, J., L. Wolfson,and L. Brown (1978) Anatomical correlates of electrical and behavioral events related to amygdaloid kindling. Ann. Neurol. 3: 538-544.

Gerfen, C. R., W. A. Staines, G. W. Arbuthnott, and H. C. Fibiger (1982) Crossed connections of the substantia nigra in the rat. J. Comp. Neurol. 207: 283-303.

Goddard, G. V., D. C. McIntyre, and C. K. Leech (1969) A permanent change in brain function resulting from daily electrical stimulation. Exp. Neurol. 25: 295-330.

Hayashi, T. (1953a) A physiological study of epileptic seizures following cortical stimulation in animals and its application to human clinics. Jpn. J. Physiol. 3: 46-54.

Hayashi, T. (1953b) The efferent pathway of epileptic seizures for the face following cortical stimulation differs from that for limbs. Jpn. J. Physiol. 3: 306-321.

Iadarola, M. J., and K. Gale (1981) Cellular compartments of GABA in brain and their relationship to anticonvulsant activity. Mol. Cell. Biochem. 39: 305-330.

Iadarola, M. J., and K. Gale (1982) Substantia nigra: Site of anticonvulsant activity mediated by $\gamma$-aminobutyric acid. Science 218 : $1237-$ 1240.

Konig, J. F. R., and R. A. Klippel (1963) The Rat Brain, Williams \& Wilkins, Baltimore.

Lippert, B., B. W. Metcalf, M. J. Jung, and P. Casara (1977) 4-Aminohex-5-enoic acid, a selective catalytic inhibitor of 4-aminobutyric acid aminotransferase in mammalian brain. Eur. J. Biochem. 74: 441-445.

Loughlin, S. E., and J. H. Fallon (1983) Dopaminergic and nondopaminergic projections to amygdala from substantia nigra and ventral tegmental area. Brain Res. 262: 334338.

McNamara, J. O. (1978) Muscarinic cholinergic receptors participate in the kindling model of epilepsy. Brain Res. 154: 415-420.

McNamara, J. O., L. C. Rigsbee, and M. T. Gulloway (1983) Evidence that substantia nigra is crucial to neural network of kindled seizures. Eur. J. Pharmacol. 86: 485-486.

Myslobodsky, M. S., R. F. Ackerman, and J. Engel (1979) Effects of $\gamma$ acetylenic GABA and $\gamma$-vinyl GABA on metrazol-activated, and kindled seizures. Pharmacol. Biochem. Behav. 11: 265-271.

Nadler, J. V., D. A. Evenson, and G. J. Cuthbertson (1981) Comparative toxicity of kainic acid and other acidic amino acids toward rat hippocampal neurons. Neuroscience 6: 2505-2517.

Parent, A., A. Mackey, Y. Smith, and R. Boucher (1983) The output organization of the substantia nigra in primate as revealed by a retrograde double labeling method. Brain Res. Bull. 10: 529-537.

Penfield, W. (1952) Epileptic automatism and the centrencephalic integrating system. Res. Publ. Assoc. Res. Nerv. Ment. Dis. 30:513528 .

Penfield, W. (1969) Epilepsy, neurophysiology, and some brain mechanisms related to consciousness. In Basic Mechanisms of the Epilepsies, H. H. Jasper, A. A. Ward, and A. Pope, eds., pp. 791-806, Little, Brown, \& Co., Boston.

Racine, R. J. (1972) Modification of seizure activity by electrical stimulation. II. Motor seizure. Electroencephalogr. Clin. Neurophysiol. 32: 281-294.

Wada, J. A., and M. Sato (1974) Generalized convulsive seizures induced by daily electrical stimulation of the amygdala in cats. Neurology 24: 565-574.

Wada, J. A., T. Mizoguchi, and T. Osawa (1978) Secondarily generalized convulsive seizures induced by daily amygdaloid stimulation in rhesus monkeys. Neurology 28: 1026-1036.

Waszczak, B. L., N. Eng, and J. R. Walters (1980) Effects of muscimol and picrotoxin on single unit activity of substantia nigra neurons. Brain Res. 188: 185-197. 